\title{
In Memory of Shoshichi Kobayashi (1932-2012): A Short Biography
}

\section{by Hisashi Kobayashi}

Shoshichi Kobayashi, Professor Emeritus of Mathematics at the University of California at Berkeley since 1994, died peacefully in his sleep on August 29, 2012, at 80 years of age. He had served on the faculty at Berkeley for 50 years, and authored over 15 books on differential geometry and the history of mathematics.

Shoshichi studied at the University of Tokyo, receiving his B.S. degree in mathematics in 1953. He spent one

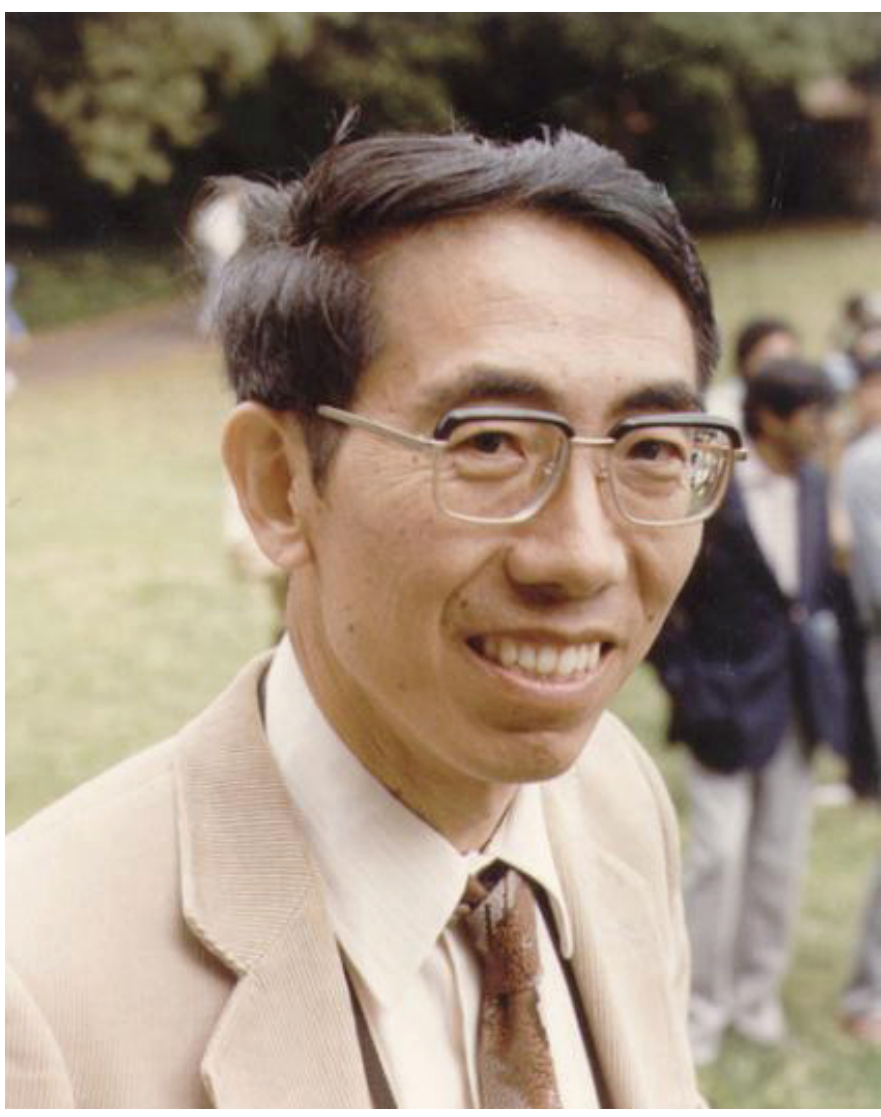

Shoshichi Kobayashi

year of graduate study in Paris and Strasbourg (1953-54) as a recipient of the French Government's scholarship, and completed his Ph.D. at the University of Washington, Seattle in 1956. He was appointed Member of the Institute for Advanced Study at Princeton (1956-58), Postdoctoral Research Associate at MIT (1958-60), and Assistant Professor at the University of British Columbia (1960-62). In 1962 he joined the faculty at Berkeley and became Full Professor in 1966. He became an emeritus professor in 1994, while remaining a professor at the Graduate School.
He was a visiting professor at numerous departments of mathematics around the world, including the University of Tokyo, the University of Mainz, the University of Bonn, MIT, and the University of Maryland. Most recently he had been visiting Keio University in Tokyo. He was a Sloan Fellow (1964-66), a Guggenheim Fellow (1977-78), and Chairman of his Department (1978-81). He was the first recipient of the Geometry Prize of the Mathematical Society of Japan established in 1987, and received an Alexander von Humboldt Prize in 1992. He was a Fellow of JSPS (Japan Society for Promotion of Science) in 1981 and 1990. Shoshichi Kobayashi was one of the most important contributors to the field of differential geometry in the last half of the twentieth century. The two-volume book Foundations of Differential Geometry coauthored with Katsumi Nomizu has had wide influence since its publication in 1963 and 1969. He published over 150 research papers.

For a complete list of his research publications, please see:

http://bibserver. berkeley.edu/cgi-bin/bibs7

?source=http: //bibserver . berkeley.edu/DB/UC

B_MATH1/Kobayashi_Shoshichi . bib

Shoshichi was also a prolific author of essays, tutorial articles and text books (mostly in Japanese), and his posthumous essay book Mathematicians Who Lost Their Faces - Essays in Idleness on Mathematics (in Japanese) was published by Iwanami Publisher in July 2013.

For details of Shoshichi's life and work and the numerous remembrance speeches and memoirs, please visit

http://en.wikipedia.org/wiki/Shoshichi_Koba yashi

or the Shoshichi Kobayashi memorial website:

www. ShoshichiKobayashi.com

The Shoshichi Kobayashi Memorial Fund was established in the Mathematics Department of Berkeley with the purpose of supporting foreign graduate students. Please visit:

www. shoshichikobayashi.com/memorial-fund 